\title{
Contextual effects for category judgments by practiced subjects'
}

\author{
ALLEN PARDUCCI AND LINDA F. PERRETT \\ UNIVERSITY OF CALIFORNIA, LOS ANGELES
}

\begin{abstract}
Category judgments of size were obtained for different sets of squares over a period of four weeks. Two Ss established fixed scales of judgment. The other two adjusted their scales to the particular set presented in each experimental session. These adjustments were consistent with a range-frequency model.
\end{abstract}

Models for the range-frequency theory treat absolute category judgments, like "large" and "small," as a compromise between two principles: (1) categories divide the psychological range of stimuli into subranges that depend only upon the endpoints of this range, and (2) categories are used with equal frequency. From these principles, the models predict the effects of manipulating relative frequencies and spacing of the stimuli presented for judgment. Previous tests have employed groups of naive Ss, each group judging a different set of stimuli (Parducci, 1963, 1965; Parducci, Calfee, Marshall, \& Davidson, 1960). The present study tests the theory on individual Ss, each of whom judges all of the sets.

\section{Method}

Four undergraduate males were paid to serve for 36 half hour sessions. The stimuli were 24 black squares. These varied in width from $1 / 2$ to $14 \mathrm{in}$. when projected on a lighted $29 \times 42$ in. screen which was approximately $10 \mathrm{ft}$ in front of the four Ss. The 24 sizes formed a geometrical progression, the width of each square being 1.16 times the width of the next smaller square. All 24 had previously been presented in one session to some 30 naive Ss, whose judgments were then used to scale the stimuli by the Thurstone method of successive intervals.

Six sets from these 24 were used in the present experiment. Each set was composed of nine different sizes with the Thurstone values represented on the abscissas of Fig. 1a, 1b, and 1c. An experimental session included 36 presentations, in random order, of each of the sizes from one of the sets. It should be noted that it is the spacing of the stimulus values that varies from set to set: In the previous tests of the range-frequency theory with psychophysical stimuli, the relative frequencies but not the spacing of the stimuli were varied. Each set was presented in six different sessions and, insofar as possible, was, preceded once by each of the other five and itself. Two different sets were presented each day in separate sessions, 1 h apart.

Sessions began with an unjudged preview in which the nine sizes for that session were presented twice in random order. Following the preview, Ss were instructed to judge each square as it was presented, assigning it to one of nine categories of size: 1Very, Very Small; 2-Very Small; 3-Small; 4-Slightly Smaller than Average; 5-Average; 6-Slightly Larger than Average; 7-Large; 8-Very Large; and 9-Very, Very Large.... in comparison with the other squares. To make his judgment, $S$ had to insert a plug in one of nine holes before the next stimulus was presented. Stimulus duration was $5-1 / 2 \mathrm{sec}$, with $1 / 2 \mathrm{sec}$ for changing slides. At the beginning of each session, Ss were reminded to make their judgments in comparison with the other squares for that session, not with squares from earlier sessions.

\section{Results and Discussion}

Two Ss did not show contextual effects: Their judgments of each stimulus value were almost independent of the set in which it was presented. The other two shifted their scales of judgment from set to set in about the same degree as the average scales for different naive groups have been found to differ for similar sets. The shifts for the different sessions in which the same set was presented did not seem related to the particular distribution presented in the immediately preceding session.

A simplified form ${ }^{2}$ of the range-frequency model described elsewhere (Parducci, 1965) was fitted to each S's judgments. As applied to the present task, in which there are the same number of categories as stimuli, this simplified model assumes that the mean of the different judgments of a stimulus is itself a weighted mean of two values: (1) a hypothetical location of that stimulus on S's psychological range, and (2) the stimulus rank, 1 through 9, in the set presented for judgment. The rank represents what the judgment would be if $S$ used his categories with equal frequency and with the fine discrimination shown by these practiced Ss.

The model was fitted by computer for the four sets having the same end stimuli, i.e., the full range sets described in Fig. 1a and 1b. The program used both the mean judgment, $\mathrm{J}$, and the rank, $\mathrm{Rk}$, of each stimulus in each set to estimate its hypothetical value, $\mathrm{Rg}$, on S's psychological range:

$$
\mathbf{J}=\omega \mathbf{R g}+(1-\omega) \mathbf{R k} .
$$

This estimate, Rg, was made for each of 11 different weightings, $\omega$, between 0 and 1.0 in steps of 0.1 . An overall range function was then fitted for each $w$, 


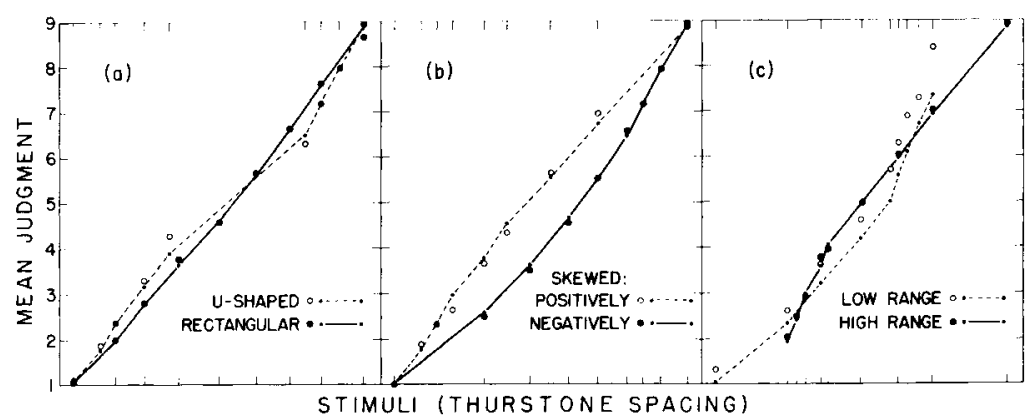

Fig. 1. Mean judgments for each set by a single S. Solid empirical points and predicted functions go with Thurstone values on the lower abscissas of the respective panels. using the Thurstone values, $X$, and the least squares criterion for the cubic function:

$$
R g=\alpha \mathbf{X}+\beta \mathbf{X}^{2}+\gamma \mathbf{X}^{3}+c
$$

Back predictions to the judgments were made by substituting the best-fitting values of $\mathrm{Rg}$ from (2) into (1) for each value of $\omega$.

The best back predictions, again by the least squares criterion, were obtained for $\omega=0.9$ for the two Ss that did not show marked contextual effects and $\omega$ $\approx 0.6$ for the other two. The 0.6 value is close to the weighting fitted for naive Ss (Parducci, 1965; Parducci et $a l, 1960)$. Figure $1 \mathrm{a}$ and $1 \mathrm{~b}$ show the predicted and obtained judgments for one of the present contextual Ss. The fits were almost as good for the other contextual S. The closeness of these fits for individual Ss gives assurance that the previous success of the range-frequency approach was not simply some artifact of averaging judgments from different Ss. It also indicates that psychophysical applications of the theory need not be restricted to effects of variation of stimulus frequencies; the theory applies just as well to the effects of stimulus spacing.

The predictions for Fig. 1c were also based on that range function and weighting that had provided the best fit for the full-range sets. The range function was almost linear, similar to the predicted meanjudgment function for the Rectangular set (Fig. 1a) but with slightly more positive acceleration. Since the inferred psychological range varied directly with the physical range of the stimuli in previous research (Parducci, 1965), it is not surprising that the predicted values are too low for the Low Range set. However, the accuracy of the predictions for the High Range set suggests that elimination of the lower stimulus values did not affect the psychological range. The width of the smallest square in this set was less than 1 in., rather close to the smallest size that could conceivably be presented in this situation. Perhaps the lower endpoint of the psychological range is anchored at this lower limit whenever the lowest stimulus value is not appreciably higher. The same interpretation would apply to the judgments by the other contextual $S$, since the relationships between his empirical and predicted judgments closely resemble those in Fig. 1c.

Separate fits of the model were obtained for the first and last of the six sessions with each of the fullrange sets. The unexpected finding was that even the two Ss that had shown contextual effects when their judgments were averaged over all sessions began with a best-fitting range weighting of 1.0 and 0.9 , respectively. Their $\omega$ values for the last sessions were 0.5 and 0.3 . Thus, the contextual effects for these practiced Ss actually increased as the experiment progressed, becoming more similar to those for naive Ss. This suggests that $S s$ require exposure to a number of different sets before establishing separate scales of judgment, at least when a large number of stimuli are presented within a single session. That half the Ss never did achieve this kind of separation between the sets might be due to the ease with which the smallest and largest squares could be anchored by the lighted background area. In further research of this kind, separation might be greater if the stimuli varied on some dimension, such as auditory loudness or pitch, for which the endpoints would be more difficult to anchor.

\section{References}

PARDUCCI, A. Range-frequency compromise in judgment. Psychol Monogr., 1963, 77, No. 2 (Whole No. 565).

PARDUCCI, A. Category judgment: a range-frequency model. Psychol. Rev., 1965, 72, 407-418.

PARDUCCI, A., CALFEE, R. C., MARSHALL, L. M., \& DAVIDSON, L. P. Context effects in judgment: Adaptation level as a function of the mean, midpoint, and median of stimuli. J. exp. PsychoL, 1960, 60, 65-77.

\section{Notes}

1. This research was supported by Public Health Service Grant HD00923, with the cooperation of the UCLA Health Sciences Computing Facility.

2. This simplified model was developed by Daniel S. Perrett. 